\section{ON GRANULAR DISEASE OF THE KIDNEYS.}

BY R. H. SEMPLE, EsQ, M.R.C.S.E.

I HAVE long believed that granular disease of the kidneys is a far more common affection than is generally supposed, and that it is really the cause of a great number of maladies, such as pleurisy, disease of the heart, \&c., which are often considered and treated as idiopathic diseases. When we consider the dreadful fatality of granular degeneration of the kidneys, and the train of secondary affections which it entails, it appears to me that the study of renal disease has been most unwarrantably neglected in this country, notwithstanding the able researches of Dr. Bright, Dr. Christison, and others; and as $I$ think it the duty of the members of our profession to add whatever information they possess to the general stock of knowledge, I am induced to offer a few remarks, illustrated with cases, upon the disease in question.

In making post-mortem examinations of persons who have died from visceral disease, it is most common to find that the kidneys deviate from the healthy structure, being sometimes larger, sometimes smaller, sometimes softer, sometimes harder; the prevailing character, however, being a deposition of granular matter, mingled with, or entirely obliterating, the cortical and tubular structure of the organ. Even this character, however, is not universal, for in the early stages the granular appearance is not perceptible, and congestion is the only morbid indication. Various as are the appearances of the kidneys after death, the symptoms during life are still more various and more obscure, the general indication being an anasarcous condition of the body, with a vagne and undefined sensation of uneasiness; or, more commonly, the disease is at first wholly latent, and its existence is only discovered after death has taken place from secondary disease. Such being the obseurity of this very common affection, it is interesting to inquire whether there exist any means of ascertaining its presence during life; and I think that we may answer the question, with tolerable certainty, in the affirmative, the albuminous condition of the urine indicating pretty clearly the existence of granular disease of the kidney. I am quite aware that albuminous urine may exist without such disease; but the causes are then transient, and the urine ceases to present the albuminons character. Hence, I make it a rule never to infer the disease from one or two examinations of the urine, but to test it repeatedly; and if I find that the albuminous deposit constantly takes place, I have very little hesitation in determining the existence of granular disease. On the other hand, I have repeatedly tested the urine of sick persons labouring under dropsy, and other diseases, and have found no albumen, and the absence of renal disease has been proved by post-mortem examination, or by the complete and permanent recovery of the patient. The discovery of the albumen in the urine is not difficult; a small test-tube and a spirit-lamp will suffice to produce a coagulum, if albumen be present; and the addition of nitric acid to another portion of urine will produce a copious white precipitate. These two tests, taken together, are conclusive evidence of the existence of albumen, but without such tests, it is wholly impossible to form any opinion; for albuminous urine presents no appearance whatever to the eye to distinguish its character; and in the early conditions of disease, even the specific gravity is nearly the same as in the healthy'state. In Case 1, from the following cases, the existence of granular disease was suspected, but not proved, during life, owing to the short interval which intervened between the medical visit and the death of the patient. In Case 2 , the disease was not even suspected, because I did not see the patient during life; but Case 3 is an excellent example of the origin, progress, and termination, of this insidious and fatal affection.

CASE 1.-Mary L-, aged thirty-six, an inmate of the Islington Workhouse, was attacked with pleurisy about a month before the date of the present report, and recovered under the ordinary treatment. As I did not attend her myself, I am unable to state the exact nature of the symptoms. It appears, however, from her statement, that she never became quite well, haviurg suffered erer since with a distressing congh. She has not menstruated for the last four or five months. She was brought into the infirmary on the 24 th of April, 1846, with all the appearances of serions disease.

Present symptoms.-Her countenance is pale, and indicative of great suffering; the lips are livid; she has great difficulty of breathing, and is unable to lie in the recumbent posture. The abdomen is swelled, the feet are cold, and the ankles are cedematous. She complains of a cough, which is hard and dry, great pain in the chest, especially on the right side, and inability to lie on that side. She has no sleep; makes water with difficulty; bowels freely open; tongue furred; pulse 85 but hardly perceptible.

Physical signs.-On percussing the chest, there is a remarkable difference between the two sides, the left side giving a pretty clear sound, the right giving a dull one. On placing the hand over the region of the heart, no undue pulsation can be perceived. On percussing the abdomen, a dull sound is produced, and a distinct fluctuation may be perceived. 'The liver cannot be felt below the edge of the ribs. On ausculta. tion, it is found that the respiration over nearly the whole of the left lung is puerile; but on the right side, the respiratory murmur can be heard only towards the apex of the lung; lower down, the respiration becomes bronchial, and æegophony is heard about midway down the lung. Over the lower part of this lung, no respiration whatever can be heard. On auscultation of the heart, no morbid sounds can be perceived, nor is the impulse of the heart much increased.

Diagnosis.-Pleuritic effusion on the right side; lung compressed by the fluid; tubercles at the apex; left lung tolerably healthy; heart generally healthy; effusion of fluid in the abdomen; liver not increased in size. Discase of the kidneys was also suspected; and as the patient's nrine had not been preserved, the nurse was requested to keep it for the next visit, but she died before any could be collected.

This patient was ordered to have beef-tea and common tea to drink; to apply a blister to the right side of the chest; and to take the following medicines: blue pill, five grains; opium, a quarter of a grain-a pill to be taken every four hours; nitrate of potash, twenty grains; tincture of digitalis, ten minims; tincture of squills, twenty minims; peppermintwater, one ounce; to be taken every four hours

She died the next morning, before the visit, and a postmortem examination was made twenty-three hours after death, when the following appearances presented themselves: - The head and neck were exceedingly livid; the swelling of the abdomen had subsided considerably, and the cdema of the ankles had disappeared. The interior of the head was not examined.-Chest: On removing the sternum, it was found that the cavity of the right pleura was filled with serous fluid, which was removed, and measured a quart. There were flakes of coagulable lymph floating in this fluid. The surfaces of the pleura were smooth. The lung on this side was very much compressed, but was otherwise healthy. The cavity of the left pleura contained a very small quantity of serous fluid, which was probably only a post-mortem appearance. The left lung was rather congested, but not otherwise diseased. The heart was generally healthy; the parietes of the left ventricle were slightly thickened, but the valves were all sound. $-A$ bdomen: There was a considerable quantity of serous effusion in the cavity of the abdomen, amounting to about a quart. The liver was not increased in size, but it was congested, and its cut surface presented the nutmeg appearance. The stomach and intestines were generally healthy. The kidneys were very much diseased; they were both very small and flabby, and their proper coat was very readily detached, exhibiting a highly granular appearance on the surface of the kidney. The same granular appearance was exhibited upon making an incision into the substance of the kidney; and, indeed, this granular structure occupied nearly the whole of both kidneys, obliterating the tubular appearance, and presenting one uniform mottled structure. The bladder contained a small quantity of urine, which, upon the application of heat, exhibited flakes of coagulated albumen.

CASE 2.- Hannah $\mathrm{W}-$, aged fifty-six, a thin, emaciated woman, who had suffered for a long time from cough and difficulty of breathing, but not to such a degree as to prevent her altogether from following her usual avocations. She had not applied for medical advice during her illness till Wednesday, June 3, 1846, when a lady, who took an interest in her case, procured the attendance of a medical gentleman. When he saw her, late at night, he found her labouring under symptoms of bronchitis, with want of sleep, difficulty of breathing, and cough; she had also some difficulty in passing her water, and the bowels were confined. She did not, however, appear to be dangerously ill, nor did she represent herself to be so. As she laboured under great debility and exhaustion, she was ordered the following:-Compound spirits of lavender, twenty minims; nitrate of potash, eight grains; sulphate of magnesia, half a drachm; infusion of senna, one ounce; to be taken every four hours. Ten grains of Dover's powder to be taken immediately. She became much better, and went to sleep; her son sleeping in the same room with her. She woke at two o'clock in the morning, and her son woke also, and gave 
her some medicine. In two hours afterwards he woke again, and found that his mother was dead. The post-mortem examination was made twenty-seven hours after death, when the following appearances presented themselves. The head was not examined.

Chest: There were a few adhesions existing between the costal and pulmonary portions of the pleura. The heart was perfectly healthy, and all the valves presented a perfectly normal appearance. The lungs did not present any remarkable appearance externally, except that the bronchial glands were somewhat enlarged, and a few deposits of calcareous matter were found at the apex of each lung. The bronchial tubes contained a large quantity of fiothy mucus; but were not remarkably thickened.-Abdomen : The liver was rather large, and its internal structure presented a nutmeg appearance, being the first stage of cirrhosis. The spleen was very small, and its structure dense. The kidneys were large, flabby, and rather pale; their investing coat was torn off rather easily, leaving the surface beneath scabrous and granular. On examining the internal structure, it was found that the right kidney presented a granular appearance in the cortical part; but the rest was pretty healthy; in the left kidney, the middle third of the organ, as well as the cortical part, were entirely converted into an homogeneous granular mass, the tubuli uriniferi in the former part being wholly absent.

As there was a little urine in the bladder, a portion of it was heated in a spoon, and a coagulum of albumen was speedily formed.

It is to be regretted that the head in this case was not examined; but the inspection took place in a very small, dark room, amidst many interruptions, and was performed necessarily in a rather hasty manner.

CASE 3.-William G-, aged forty-six, formerly a teacher of the French language, and mathematics, was admitted into the Infirmary of the Islington Workhouse on the 26th of December, 1846. This poor man states that he has undergone a series of misfortunes; but denies having ever been addicted to drinking, although he admits that he used to drink a glass or two of spirits every day while residing in Guernsey, where liquors are very cheap; but that for the last two years he has ceased drinking spirits altogether. Having latterly become greatly reduced in circumstances, so as to be unable to procure the common necessaries of life, he has been exposed to wet, cold, and hunger, being often compelled to sleep in the streets. About a month ago, after continued exposure to wet and cold, he perceived that his legs began to swell, and at the same time he observed that his perspiration was checked, and that the secretion of urine was very scanty. At that time, he did not suffer any pain; but only experienced a general feeling of languor and listlessness; but since then, he has suffered considerable pain in the back, loins, and shoulders. He applied for admission into St. Bartholomew's Hospital; but could not be admitted, as the hospital was full; and he was therefore sent into the Islington Infirmary.

Present state- - He presents a bloated and anasarcous ap. pearance, the legs, scrotum, and face, being swollen; the cedematous parts are pitted on pressure. He suffers oceasional pain in the neck, and experiences a sensation of tightness about his limbs. Tongue tolerably clean as to colour, but dry and rough; great thirst; appetite pretty good; skin dry and harsh; no perspiration; pulse 100, very weak, bu regular; no pain on pressure in any part of the body. He complains of occasional congh; but without any difficulty of breathing. There is no morbid sound whatever either in the heart or the lungs, when those parts are examined by percussion and auscultation. The urine is now rather copious and clear. At this visit, a small quantity of nitric acid was added to a portion of urine, and a copious precipitate was formed. As the other chemical tests were not in readiness, the farther examination of the urine was deferred until the next day, and in the meantime, a little saline medicine was administered, containing small doses of tincture of henbane. The bowels are open, and he sleeps pretty well.

Dec. 27th.-Much the same. The urine was subjected to the action of heat, but did not give any coagulum-a circumstance I find it difficult to account for, as on many subsequent occasions a dense coagulum was produced. Nitric acid again gave a copious white precipitate; nitrate of silver gave a curdy precipitate; sulphate of copper gave a light-blue one; corrosive sublimate a white one; and ferrocyanuret of potassium, acidulated with a little vinegar, gave a whitish precipitate. To avoid unnecessary repetition, it may be stated that the urine was frequently tested on subsequent occasions, and always with the same results. The diagnosis was granular disease of the kidneys, and the following treatment was adopted:- He was directed to be kept warm by additional blankets; to have full diet; to drink as much as he pleased of tea and cream-of-tartar water, and to take the following:Dover's powder, fifteen grains, to be taken every night. Tincture of henbane, spirits of nitric ether, of cach twenty minims; nitrate of potash, twenty grains; solution of acetate of ammonia, camphor mixture, of each half an ounce; to be taken every four hours.

29th. - Is rather better; has passed a good night; tongue moist and soft; has had, for the first time, profuse perspiration; urine as before. To continue the medicines.

Jan. 2nd.-Pulse full; tension of the skin; pain across the back and loins; urine still exhibiting a highly albuminous character; bowels confined. In order to relieve the pain and tension, a small bleeding was ordered, but only a few ounces of blood could be drawn. To continue the medicines, and to take, in addition, one scruple of jalap, and two scruples of cream-of-tartar, twice a day.

4th. - Has perspired freely, and the tension of the skin has disappeared; feels altogether better. To continue the medicines.

6th.-Still continues to improve. The improvement went on gradually; he passed good nights; his urine was copious, and his perspiration was abundant; the cedema was daily diminishing; his tongue was moist and clean, and he relished his food. Thus he continued till Jan. 21st, when his pulse rose to 168, and he felt generally uneasy, but without describing any particular symptoms.

23rd.-Says he feels very ill and low-spirited. The pulse is 144; he has a hard cough, and pain in the chest, with a little gluey expectoration. On examination by auscultation, the right lung gave a crepitant rattle throughout. Diagnosis: Pneumonia of the right lung. To apply a blister to the chest, and to take the following: calomel, two grains; opium, onethird of a grain, every four hours; tartarized antimony, onethird of a grain; tartrate of potash, twenty grains; liquor of acetate of ammonia, and camphor mixture, of each half an ounce, every four hours.

$24 \mathrm{th}$-DLuch worse; pain in the chest, cough, and difficulty of breathing, all increased; blister has not risen; pulse 152 respirations 40 in a minute; tongue dry, rough, and furred; sordes about the lips, teeth, and gums; very low-spirited, but intellectual faculties entire; urine gives a copious precipitate when heated.

He died on the morning of the 25 th of January. A postmortem examination was made twenty-four hours after death, when the following appearances were observed. The body was generally anasarcous.

Head : The scalp and skull presented no unusual appearances. On removing the latter, and the dura mater, the arachnoid membrane presented an opaque pearly appearance. This membrane was much thickened, and a considerable quantity of serous fluid was effused beneath it.

Chest.-There was a large quantity of serous fluid in the cavity of each pleura, and also in the pericardium. The heart was healthy throughout, and all its valves were perfectly sound. The surface of the pulmonary portion of the right pleura was partially covered with a layer of recent coagulable lymph, of about the thickness of ordinary leather, easily detached from the subjacent pleura. Shreds and patches of the same coagulable lymph were scattered over other portions of the pleura, or remained floating in the serum. The upper lobe of the right lung was completely hepatized, and its cut surface exuded a dirty kind of pus. The lower lobe was much congested. The left lung was carnified, but did not exhibit appearances of recent disease.

Abdomen.-A considerable quantity of serous fluid in the cavity of the peritonceum. There were a few Hakes of coagulable lymph on the surface of the liver. This organ presented a mottled appearance on the outside, and, when cut, the incised surface presented a nutmeg appearance. The kidneys were both diseased, and as both were alike, a description of one will be sufficient. The size was much greater than was natural, by about one half; the proper membrane was readily detached; the colour was dark, and the consistence flabby; the appearance was mottled; when cut through, the cortical and tubular structure were found almost entirely absent, and their place was supplied by granular matter of a grey colour; each kidney was sliced and cut in various directions, and the same appearances were everywhere exhibited in the cortical and tubular portions; but the ureters, pelvis, and calices, presented the ordinary structure.

February, 1846 . 